\title{
Estudio de la efectividad de tres aceites esenciales para el control de áfidos en pimiento, Capsicum annuum $\mathrm{L}$.
}

\author{
Study of the effectiveness of three essential oils \\ to control aphids on pepper plants Capsicum annuum $L$. \\ Jorge Eduardo Castresan ${ }^{1}$, Javier Rosenbaum ${ }^{1}$, Laura Alicia González ${ }^{3}$
}

\section{RESUMEN}

El pulgón es una plaga muy común en cultivos hortícolas siendo relevantes tres especies: Aphis gossypii (Glover), Myzus persicae (Sulzer) y Macrosiphum euphorbiae (Thomas) (Hemíptera: Aphididae). Los pulgones producen importantes pérdidas económicas, debidas a su periódica aparición y al daño que ocasionan, tanto por la extracción de savia, como por la transmisión de enfermedades virósicas, principalmente en cultivos hortícolas. Algunos aceites esenciales extraídos de ciertas plantas tienen propiedades insecticidas y acaricidas de amplio espectro sobre artrópodos de cuerpo blando, esto hace posible utilizarlos como fuente alternativa para el manejo integrado de plagas. El presente trabajo tuvo como objetivo evaluar la efectividad repelente de tres distintos aceites esenciales Eucaliptus globulus Labill., Rosmarinus officinalis (Linn.), Allium sativum L. sobre los pulgones A. gossypii, M. persicae y M. euphorbiae, en cultivo de pimiento (Capsicum annum híbrido Paloma) en invernadero biosolarizado y no biosolarizado. Para ello se dispusieron en ambos 15 parcelas, cada una con 15 plantas de pimiento. Para evaluar los tratamientos se efectuaron 20 monitoreos totales, cada uno semanalmente, registrándose en cada parcela, sobre tres plantas seleccionadas previamente a la aplicación de aceites esenciales, el número promedio de pulgones totales (adultos ápteros + alados + ninfas) y de pulgones parasitados (momias) en el envés de cuatro hojas del estrato medio de la planta. Los datos analizados con un ANAVA para un DCA con tres repeticiones. Los resultados mostraron que tratamientos con aceite esencial de ajo (A. sativum) + aceite vegetal de soja y aceite esencial de Eucalipto (E. globulus) + aceite vegetal de soja modificaron el comportamiento de los áfidos, registrándose un menor número de individuos.

Palabras clave: áfidos, aceites esenciales, eucaliptus, romero, ajo, pimiento.

\section{SUMMARY}

Aphid is a very common pest in horticultural crops and the three most important pests are: Aphis gossypii (Glover), Myzus persicae (Sulzer) and Macrosiphum euphorbiae (Thomas) (Hemíptera: Aphididae). Aphid causes severe economic losses due to its periodic occurrence and damage caused by both sucking sap from plants and spreading plants diseases through the transmission of viruses, mainly, in horticultural crops. Some essential oils extracted from certain plants have broad-spectrum insecticidal and acaricidal properties on soft-bodied insects; therefore, they may be used as an alternative source for an integrated pest management. The main purpose of this work was to evaluate the repellent effectiveness of various essential oils "treatments", such as Eucalyptus globulus Labill., Rosmarinus officinalis (Linn.), Allium sativum L. on aphids A. gossypii, M. persicae and M. euphorbiae in pepper crops (Hybrid Pepper "Paloma" Capsicum annuum), in greenhouses with or without biosolarization. To achieve this goal, 15 parcels with 15 peppers plants each were arranged in each of these greenhouses. The trial consisted of weekly individual monitoring (20 times in total) and the average number of total aphids (wingless adults + winged aphids + nymphs) and parasitized aphids (mummies) on the underside of 4 medium leaves on 3 plants selected prior to the application of essential oils in each parcel was reported. Each treatment was replicated three (3) times and the data was analyzed using an Analysis of Variance (ANOVA) for a Completely Randomized Design. The trials proved that treatments with garlic essential oils (A. sativum) + soybean oil and Eucalyptus essential oil (E. globulus) + soybean oil modify aphids' behavior resulting in a lower number of these insects.

Key words: aphids, essential oils, eucalyptus, rosemary, garlic, pepper.

1 Estación Experimental Agropecuaria INTA Concordia, C.P. 3200, Estación Yuquerí, Concordia, Entre Ríos, Argentina (Tel/ Fax: 5403454290000 / 4290215.

2 Universidad Nacional de Córdoba.

* Autor para correspondencia: jcastresana@ correo.inta.gov.ar

Fecha de Recepción: 19 Marzo, 2013.

Fecha de Aceptación: 8 Julio, 2013. 


\section{Introducción}

Los áfidos son importantes plagas que afectan el cultivo de pimiento protegido (Valério et al., 2005). Dentro de estos, entre las especies más importantes mencionadas se encuentran: A. gossypii (Glover), (pulgón del algodón), M. persicae Sulzer (pulgón verde del duraznero) (Vasicek, et al., 2001) y M. euphorbia Thomas (pulgón verde de la papa). El ambiente particular de los invernaderos brinda las condiciones óptimas para el desarrollo de las poblaciones de dichos áfidos. Estos insectos ocasionan dos tipos de daño: 1) directo, provocado por la succión de fotosintatos por adultos y ninfas y 2 ) indirecto, debido a que las ninfas eliminan sustancias ricas en hidratos de carbono sobre las que se desarrollan gran cantidad de hongos, conocidos vulgarmente como fumagina (Cabello \& Belda, 1994). A este daño indirecto debe agregarse otro más grave aún, que consiste en la capacidad de algunos áfidos de comportarse como vectores de virus (Syller, 1994). M. persicae, A. gossypii y M. euphorbia, entre otros han sido detectados transmitiendo diferentes virus que atacan al pimiento, con excepción de TMV y PVX que se transmiten solo por contacto (Smith, 1972). Aunque los mayores perjuicios son causados por el "Pulgón verde del duraznero" siendo sin lugar a dudas la transmisión de fitovirus el problema más importante. Es considerado por muchos autores como el más importante vector en el mundo, habiéndose registrado la transmisión de más de 100 virus por esta especie; algunas enfermedades particularmente dañinas incluyendo al Potato leafroll virus (PLRV) y el virus Y de la papa (PVY) en Solanáceas (Capinera, 2005). Otros virus determinados en pimiento y transmitidos eficientemente por M. persicae son el Mosaico del pepino (CMV) y el Mosaico de la alfalfa (AMV). Se utilizan numerosos insecticidas sintéticos para el control de los áfidos siendo M. persicae una de las plagas más estudiadas en las últimas décadas. Debido a que ha originado distintos mecanismos de resistencia a insecticidas, ha sido registrada en 31 países y se han utilizado para su control un total de 69 insecticidas dentro de los fosforados, clorados, carbamatos y piretroides (Fuentes et al., 2007), lo mismo ha sucedido con A. gossypii en la mayoría de las áreas de cultivo de algodón en el mundo entero. (Herron et al., 2001). Algunos aceites esenciales derivados de ciertas plantas tienen tanto propiedades insecticidas como acaricidas de amplio espectro sobre insectos de cuerpo blando (Isman, 1999) y además son altamente lipofílicos, por lo cual penetran fácilmente en la cutícula del insecto. Esto hace posible utilizarlos como fuente alternativa de control. Los aceites esenciales pueden inhibir la respiración, disminuir la alimentación, afectar el crecimiento, reducir la fecundidad, provocar la disrupción de la cutícula y la actividad sobre el camino de la octopamina en el sistema nervioso central (Akhtar \& Isman, 2004). Los aceites esenciales derivados de las plantas tienen un poder de actividad residual corto, con intervalos menores a 12 horas, siendo susceptibles a la degradación por altas temperaturas y luz ultravioleta (Miresmailli \& Isman, 2006). Los aceites esenciales son efectivos controlando el pulgón del algodonero (A. gossypii) y el pulgón verde del duraznero M. persicae (Isman, 1999). Algunos aceites esenciales están disponibles para los consumidores sin haber sido evaluados previamente en el control de plagas (Trumble, 2002). Básicamente contienen monoterpenos (C10) (constituyen aproximadamente el $90 \%$ de las mezclas) y sesquiterpenos (C15), y una variedad de fenoles 23 aromáticos, óxidos, éteres, alcoholes, ésteres, aldehídos y cetonas que determinan el aroma y bioactividad característicos de la planta de la cual provienen (Batish et al., 2008). En el caso del aceite esencial de romero $(R$. officinalis: Laminaceae) la toxicidad fumigante de su aceite esencial es altamente efectiva contra Tribolium castaneum Hbst. (Ahmadi et al., 2007) y Acanthoscelides obtectus (Say) (Regnault-Roger et al., 2004), debido a la presencia de 1,8-cineol y alcanfor (Zaouali et al., 2010). Los vapores aromáticos del romero tienen efecto ovicida sobre el ácaro arañuela roja (Tetranychus urticae Koch). Además este puede tener efectos subletales, actuando como repelente del trips de la cebolla (Thrips tabaci Lindeman) (Sedy \& Koschier, 2003). Con respecto al aceite esencial de Eucalipto blanco (E. globulus: Myrtaceae), el compuesto más abundante es 1,8-cineol, conocido vulgarmente como eucalyptol, o simplemente como cineol. Sin embargo este compuesto no resultó ser toxico para larvas del mosquito Aedes aegypti Linn. mientras que los restantes componentes como el isotiocianato de alilo, (E)-nerolidol, limoneno, p-cimeno e y-terpineno mostraron una fuerte actividad larvicida contra A. aegypti (Park et al., 2011). Hay además otros trabajos que demuestran la actividad repelente del aceite esencial de eucaliptus y sus principales componentes para diferentes especies de áfidos M. persicae y Brevycorine brassicae (Ricci et al., 2010). Por último, el aceite esencial de ajo 
(Allium sativum: Liliaceae) tiene dos componentes principales, disulfuro de metilo alilico y dialilo trisulfuro, ambos tienen una alta toxicidad contra Sitophilus zeamais (Mostsch.) y T. castaneum (Ho et al., 1996). Más recientemente, (Hori, 1996) demostró que el aceite esencial de ajo fue tóxico para M. persicae evitando que los áfidos se establezcan. El objetivo del presente estudio fue determinar la efectividad repelente de los aceites esenciales de romero, eucalipto y ajo para A. gossypii, M. persicae y M. euphorbia en cultivo de pimiento (Capsicum annиuт L) en invernadero biosolarizado y no biosolarizado.

\section{Materiales y Métodos}

Los estudios sobre el efecto repelente de los aceites esenciales sobre los áfidos fueron llevados a cabo en dos invernaderos pertenecientes al módulo hortícola de la Estación Experimental INTA Concordia, ubicado en la localidad de Concordia, Entre Ríos, Argentina ( $31^{\circ} 22^{\prime} 22.16^{\prime \prime}$ LS; $58^{\circ}$ 7'5.42" LO) y una altitud de $396 \mathrm{msnm}$ ). Se usaron dos módulos de $240 \mathrm{~m}^{2}$ de superficie cubiertos con filme de polietileno de 100 micrones. Luego de un cultivo invernal de acelga, en ambos invernáculos, se incorporaron ocho $\mathrm{kg}$ cama de pollo/ $\mathrm{m}^{2}$ previamente a la plantación del cultivo de pimiento. Solamente en uno de los invernaderos se realizó la biodesinfección con solarización (biosolarización), la cual ha demostrado ser un método eficaz para regular las poblaciones de nematodos, patógenos fúngicos y malas hierbas. La biofumigación con solarización realizada en forma reiterada no solo no tiene efectos negativos sobre el suelo, sino que, en comparación con suelos sin aporte de materia orgánica, revitaliza la actividad microbiana y los ciclos biogeoquímicos. Siendo eficaz todo ello cuando se incluye dentro de un programa de manejo integrado de los sistemas de producción con el propósito de enmarcar este cultivo hacia la agricultura ecológica.
La fertilización fue igual en ambos invernaderos, iniciándose luego de la primera floración con una periodicidad de 15 días por riego, para satisfacer las demandas del cultivo, como así también las restantes labores como la eliminación de malezas, hilado y corte de frutos. La siembra se realizó en abril de 2010 con semillas de pimiento dulce (C. апnиит) híbrido Paloma de la empresa Seminis, en mesas de germinación. Una vez que las plantas tuvieron dos cotiledones desarrollados se mantuvieron en el semillero hasta que alcanzaron aproximadamente $15 \mathrm{~cm}$ de altura con desarrollo adecuado para su plantación, en mayo de 2010. Durante los cinco meses en los que el cultivo estuvo en el invernadero, las únicas plagas de importancia fueron los áfidos A. gossypii y M. euphorbiae. Para evaluar este ensayo sobre pulgones se utilizaron diferentes aceites esenciales obtenidos del laboratorio EUMA S.A. (Villa Adelina, San Isidro, Buenos Aires, Argentina). En ambos invernaderos, sobre tres camellones $0,50 \mathrm{~m}$ de ancho por $28 \mathrm{~m}$ de largo, se delimitaron en cinco parcelas y en cada una de ellas se dispusieron 15 plantas de pimiento. La asignación de los tratamientos (Tabla 1) a las parcelas fue aleatoria. Los datos se analizaron con un ANVA para un diseño completamente aleatorizado con tres repeticiones. Las aplicaciones de los tratamientos se realizaron semanalmente de junio a octubre de 2010 efectuándose con diferentes mochilas manuales (pulverizador dorsal simétrico SP marca Guarany), equipadas con boquilla cónica, a una presión máxima de $500 \mathrm{kpa}$ (75 psi) y volumen de agua de 20 litros. Para evaluar los tratamientos se efectuaron 20 monitoreos totales, cada uno semanalmente, registrándose en cada parcela, sobre tres plantas seleccionadas previamente a la aplicación de aceites esenciales, el número promedio de pulgones totales (adultos ápteros + alados + ninfas) y de pulgones parasitados (momias) en el envés de cuatro hojas del estrato medio de la planta según protocolo (Polack $\&$ Mitidieri, 2002).

Tabla 1. Aceites esenciales y dosis evaluadas.

\begin{tabular}{clccc}
\hline Tratamiento & \multicolumn{1}{c}{ Aceite esencial } & Dosis $(\%)$ & Coadyuvante & $\%$ \\
\hline 1 & Eucaliptus globulus & 0,5 & Aceite vegetal de soja & 0,3 \\
2 & Rosmarinus officinalis & 0,5 & Aceite vegetal de soja & 0,3 \\
3 & Allium sativum & 0,125 & Aceite vegetal de soja & 0,3 \\
4 & Aceite vegetal de soja & 0,3 & & \\
5 & Control (agua) & & & \\
\hline
\end{tabular}




\section{Resultados}

Los datos fueron analizados estadísticamente usando el software estadístico InfoStat. Mediante un modelo donde se utilizó la transformación raíz cuadrada de los valores de la variable +1 , con el fin de normalizar los resultados. La parcela se incluyó en el modelo como un efecto aleatorio. Además se corrigió la heterocedasticidad usando herramientas del menú de modelos mixtos del programa, realizando un análisis por separado para cada invernadero.

\section{Variable número de áfidos promedio}

En las fechas de monitoreo 19/08/2010, $23 / 08 / 2010$ y $01 / 09 / 2010$ en el invernadero biosolarizado se produce un aumento en el número promedio de áfidos (ninfas + adultos), sobre todo en la fecha 23/08/2010. En las mismas, los tratamientos control (pulverización con agua) y aceite vegetal de soja (Glycine max) registran un incremento en el número de áfidos que los restantes tratamientos, mientras que el de aceite esencial de ajo (Allium sativum) + aceite vegetal de soja y aceite esencial de romero (Rosmarinus officinalis) + aceite vegetal de soja (G. max) son estadísticamente iguales, siendo el tratamiento aceite esencial de eucalipto blanco (Eucalyptus globulus) + aceite vegetal de soja el que mejor controla el número de pulgones (Figura 1). En las fechas de monitoreo 12/08/10, 19/08/10, 23/08/10 y 01/09/10 en el invernadero no biosolarizado se produce un aumento en el número promedio de áfidos (ninfas + adultos), acentuado en la fecha 23/08/10. En la misma se observa que los tratamientos control (pulverización con agua) y aceite vegetal de soja (G. max) registran un incremento en el número de áfidos que los restantes tratamientos, mientras que el de aceite esencial de romero ( $R$. officinalis) tuvo un comportamiento intermedio y finalmente ambos tratamientos de aceite de ajo (A. sativum) y aceite de eucalipto (E. globulus) son estadísticamente iguales, resultando estos los que mejor controlan el número de áfidos (ninfas + adultos) (Figura 2).

\section{Variable número promedio de áfidos parasitados}

Entre las fechas de monitoreo 23/08/10 y 13/10/10 en el invernadero biosolarizado se produce un aumento en el número promedio de áfidos parasitados por Aphidius colemani (Haliday) y Diaeretiella rapae (McIntosh). Sin embargo, respecto del análisis no se detectó interacción significativa entre tratamiento y semana como tampoco un efecto del tratamiento. Teniendo solo la semana un efecto significativo (Figura 3). Entre las fechas de monitoreo 23/08/10, 07/09/10 y 22/09/10 en el invernadero no biosolarizado se produce un aumento en el número promedio de áfidos parasitados por (A. colemani y D. rapae). Sin embargo, no se detectó interacción significativa entre tratamiento y semana y tampoco un efecto del tratamiento. Solo se encontró que la semana tuvo un efecto significativo (Figura 4).

\section{Variable peso promedio para distintos invernaderos}

Los datos analizados con relación al peso promedio de frutos de pimiento registraron un efecto de invernáculo $(P<0,05)$, con lo cual se observa que los pesos promedios mayores se obtienen en el invernáculo biosolarizado respecto del no biosolarizado.

Para el invernadero biosolarizado (Figura 5) hay un efecto de tiempo $(P<0,05)$. Con lo cual se observa que el tratamiento aceite esencial de ajo (A. sativum) + aceite vegetal de soja afecta menos al peso promedio de los frutos de pimiento. Sin embargo, en la cosecha $\mathrm{N}^{\circ} 3$ este tratamiento difiere estadísticamente de los demás. No diferenciándose entre sí para los restantes tratamientos y pesos promedio de fruto en las distintas cosechas realizadas. Para el invernadero no biosolarizado (Figura 6) hay un efecto de tiempo $(P<0,05)$. Observándose que el tratamiento aceite esencial de ajo (A. sativum) + aceite de soja (G. max) afecta menos al peso promedio de los frutos de pimiento; sin embargo, para cada cosecha no se detectaron diferencias estadísticamente significativas con los demás tratamientos. Sí diferenciándose la cosecha $\mathrm{N}^{\mathrm{o}} 1$ de la $\mathrm{N}^{\mathrm{o}} 2$ y de la $\mathrm{N}^{\mathrm{o}} 3$.

\section{Discusión y Conclusión}

Los resultados en el presente estudio incluyen observaciones de diferencias en comportamiento de distintos áfidos M. euphorbiae y A. gossypii sobre la elección como hospedero de plantas de pimiento en el tiempo, luego de ser tratadas con distintos aceites esenciales. Esto sugiere que diferentes 
INVERNADERO BIOSOLARIZADO

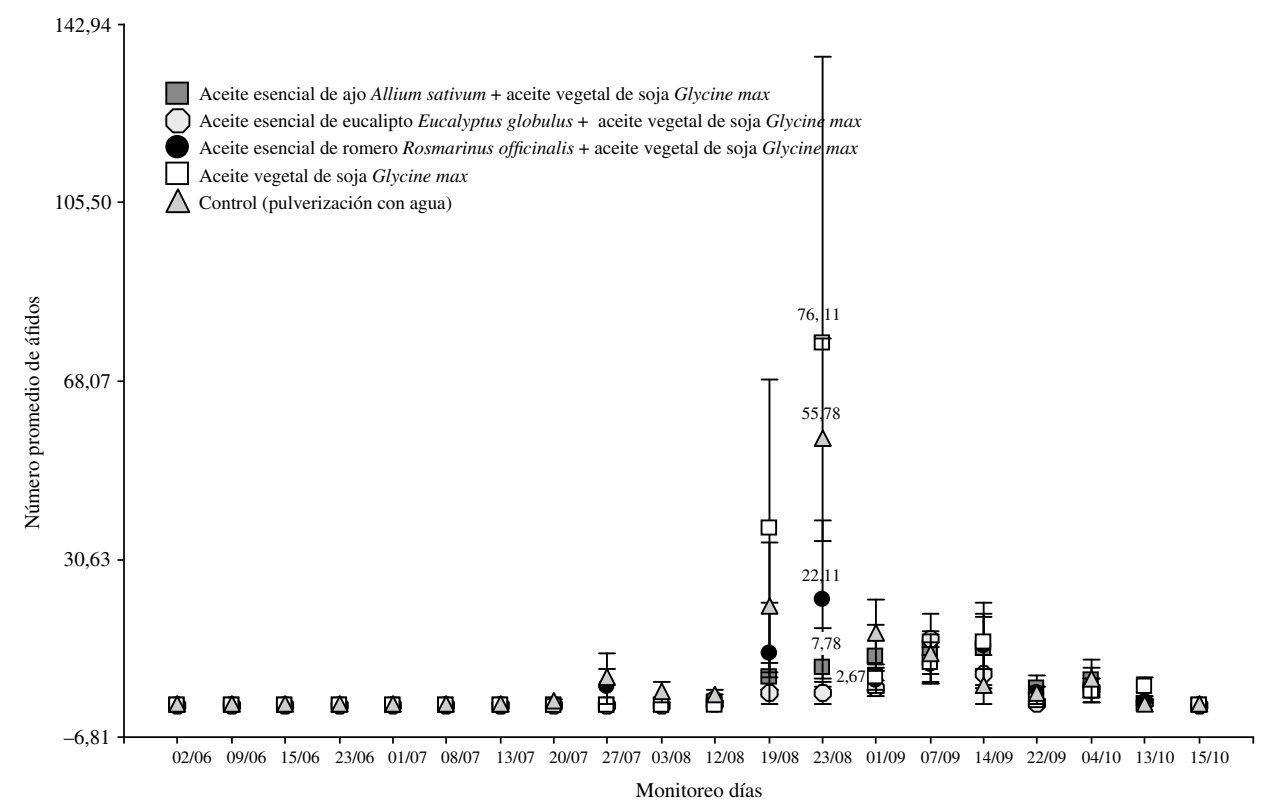

Figura 1. Población de áfidos (ninfas + adultos) sobre distintos tratamientos en invernadero biosolarizado.

Medias ajustadas y errores estándares para Tratamiento * Semana

$\operatorname{DGC}($ Alfa $=0,05)$

\begin{tabular}{lrrrrrr}
\hline \multicolumn{1}{r}{ Tratamiento } & Fecha & Medias & \multicolumn{1}{c}{ E.E. } & & \\
\hline Aceite de soja & $23 / 08$ & 76,11 & 15,15 & A & \\
Control (agua) & $23 / 08$ & 55,78 & 6,23 & A & & \\
Ac. es. romero & $23 / 08$ & 22,11 & 4,96 & & B & \\
Ac. es. ajo & $23 / 08$ & 7,78 & 2,93 & & B & \\
Ac. es. eucaliptus & $23 / 08$ & 2,67 & 1,77 & & C \\
\hline
\end{tabular}

Análisis de la varianza

\begin{tabular}{lcrrr}
\hline & numDF & denDF & F-value & \multicolumn{1}{c}{ p-value } \\
\hline (Intercept) & 1 & 190 & 281,71 & $<0,0001$ \\
Tratamiento & 4 & 10 & 1,13 & 0,3947 \\
Semana & 19 & 190 & 16,01 & $<0,0001$ \\
Tratamiento: semana & 76 & 190 & 1,44 & 0,0238
\end{tabular}

Hay interacción estadísticamente significativa entre tratamiento y semana $(\mathrm{p}=0,0238)$.

tratamientos con aceite esencial de ajo (A. sativum) + aceite vegetal de soja y aceite esencial de eucalipto (E. globulus) + aceite vegetal de soja modificaron el comportamiento de los áfidos, registrándose un menor número de individuos. Similares diferencias han sido observadas en estudio con M. persicae. Donde ambos aceites de ajo $A$. sativum y cebolla (Allium cepa $\mathrm{L}$.) favorecen la inhibición de la colonización por parte de estos áfidos y poseen actividad insecticida, pero no la actividad antialimentaria (Hori, 1996). Además de su acción insecticida, tanto el jugo de ajo picados y el extracto de acetato de etilo de ajo ha demostrado ser altamente repelente para estas dos especies de escarabajos (Ho et al., 1996), por otro lado el allyl isotiocianato como el aceite de Allium sativum incrementó la mortalidad de adultos de cucaracha alemana Blattella germanica, citado por (Tunaz et al., 2009). Con respecto al aceite esencial de Eucalyptus globulus los componentes 
INVERNADERO NO BIOSOLARIZADO

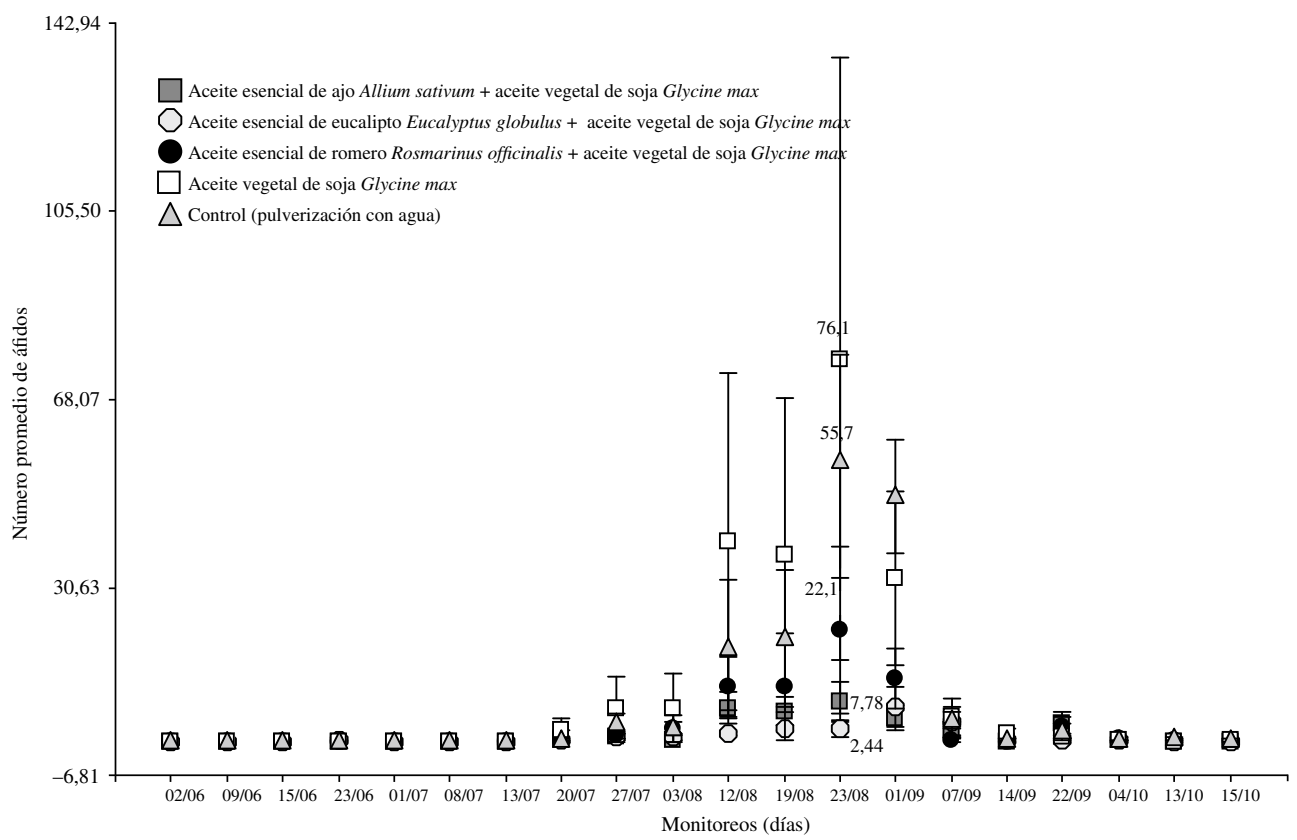

Figura 2. Población de áfidos (ninfas + adultos) sobre distintos tratamientos en invernadero no biosolarizado.

Medias ajustadas y errores estándares para Tratamiento * Semana

$\operatorname{DGC}($ Alfa=0,05)

\begin{tabular}{lcrrrrr}
\hline \multicolumn{1}{c}{ Tratamiento } & Semana & Medias & \multicolumn{1}{c}{ E.E. } & & \\
\hline Aceite de soja & 13 & 76,11 & 17,37 & A $\square$ & \\
Control agua & 13 & 55,78 & 6,93 & A $\square$ & \\
Ac. es. romero & 13 & 22,11 & 4,72 & & B & \\
Ac. es. ajo & 13 & 7,78 & 1,55 & & C \\
Ac. es. eucalipto & 13 & 2,44 & 1,39 & & C \\
\hline
\end{tabular}

Análisis de la varianza

\begin{tabular}{lccrr}
\hline & numDF & denDF & F-value & p-value \\
\hline (Intercept) & 1 & 190 & 215,12 & $<0,0001$ \\
Tratamiento & 4 & 10 & 2,45 & 0,1146 \\
Semana & 19 & 190 & 14,66 & $<0,0001$ \\
Tratamiento: semana & 76 & 190 & 2,05 & $<0,0001$ \\
\hline
\end{tabular}

Hay interacción estadísticamente significativa entre tratamiento y semana $(\mathrm{p}=<0,0001)$.

allyl isotiocianato, (E) -nerolidol, limoneno, $p$-cymeno e $y$ - terpineno demostraron fuerte actividad larvicida contra Aedes aegypti (Park et al., 2011). Como también (Regnault-Roger et al., 2004) demostró que el aceite esencial de Eucalyptus globulus tiene actividad adulticida, ovicida y larvicida contra la plaga de grano almacenado Acanthoscelides obtectus (Coleoptera: Bruchidae). Por último cabe aclarar que el aceite esencial de romero ( $R$. officinalis) en nuestro caso no fue tan eficiente como los anteriores aceites esenciales, como demuestran varias citas bibliográficas, donde muestra tener fuerte efectos adversos sobre el desempeño reproductivo del áfido de la col (Işık \& Görür, 2009) y puede ser considerado como un acaricida contra la arañuela roja Tetranychus urticae (Acari: Tetranychidae) causando mortalidad completa en el laboratorio con concentraciones que 
INVERNÁCULO BIOSOLARIZADO

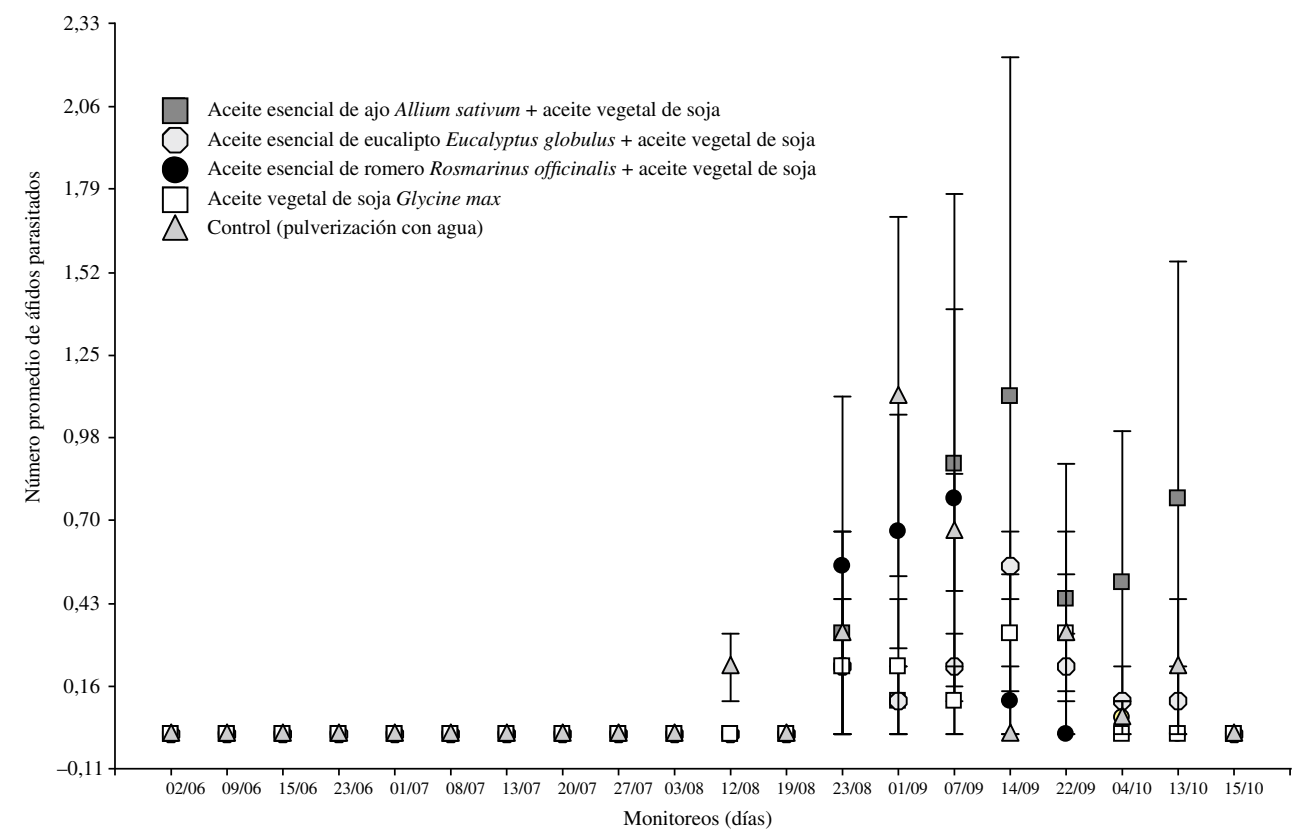

Figura 3. Población de áfidos parasitados sobre distintos tratamientos en invernadero biosolarizado.

Análisis de la varianza

\begin{tabular}{lrrrr}
\hline & numDF & denDF & F-value & p-value \\
\hline (Intercept) & 1 & 190 & 31872,08 & $<0,0001$ \\
Tratamiento & 4 & 10 & 1,18 & 0,3763 \\
Semana & 19 & 190 & 5,89 & $<0,0001$ \\
Tratamiento: semana & 76 & 190 & 0,87 & 0,7610 \\
\hline
\end{tabular}

Del análisis realizado se determinó que no hay interacción estadísticamente significativa entre tratamiento y semana $(\mathrm{p}=0,7610)$, tampoco diferencias entre tratamientos $(\mathrm{p}=0,3763)$; sin embargo, existe efecto del tiempo $(\mathrm{p}=<0,0001)$.

no causan fitotoxicidad a las plantas hospederas (Miresmailli \& Isman, 2006). El aceite puede tener efecto subletal como acción repelente sobre trips de la cebolla Thips tabaci Lindeman (Sedy \& Koschier, 2003) (Los aceites esenciales usados en la presente investigación son elaborados y desarrollados como saborizantes de alimentos, golosinas, bebidas y medicamentos. Como también para la industria cosmética y perfumería. Por lo tanto, son considerados no nocivos o menos para la salud humana que la mayoría de los pesticidas convencionales (Topuz \& Erler, 2007). Teniendo en cuenta los resultados obtenidos en este ensayo se observó que la fauna benéfica aparece a pesar de las aplicaciones semanales con los distintos aceites esenciales.

Esto exige la necesidad de seguir investigando con la finalidad de diseñar un método de producción alternativo y sustentable a la aplicación de insecticidas sintéticos para el control de plagas, minimizando los daños que pudieran ocasionar estos a la fauna benéfica. 


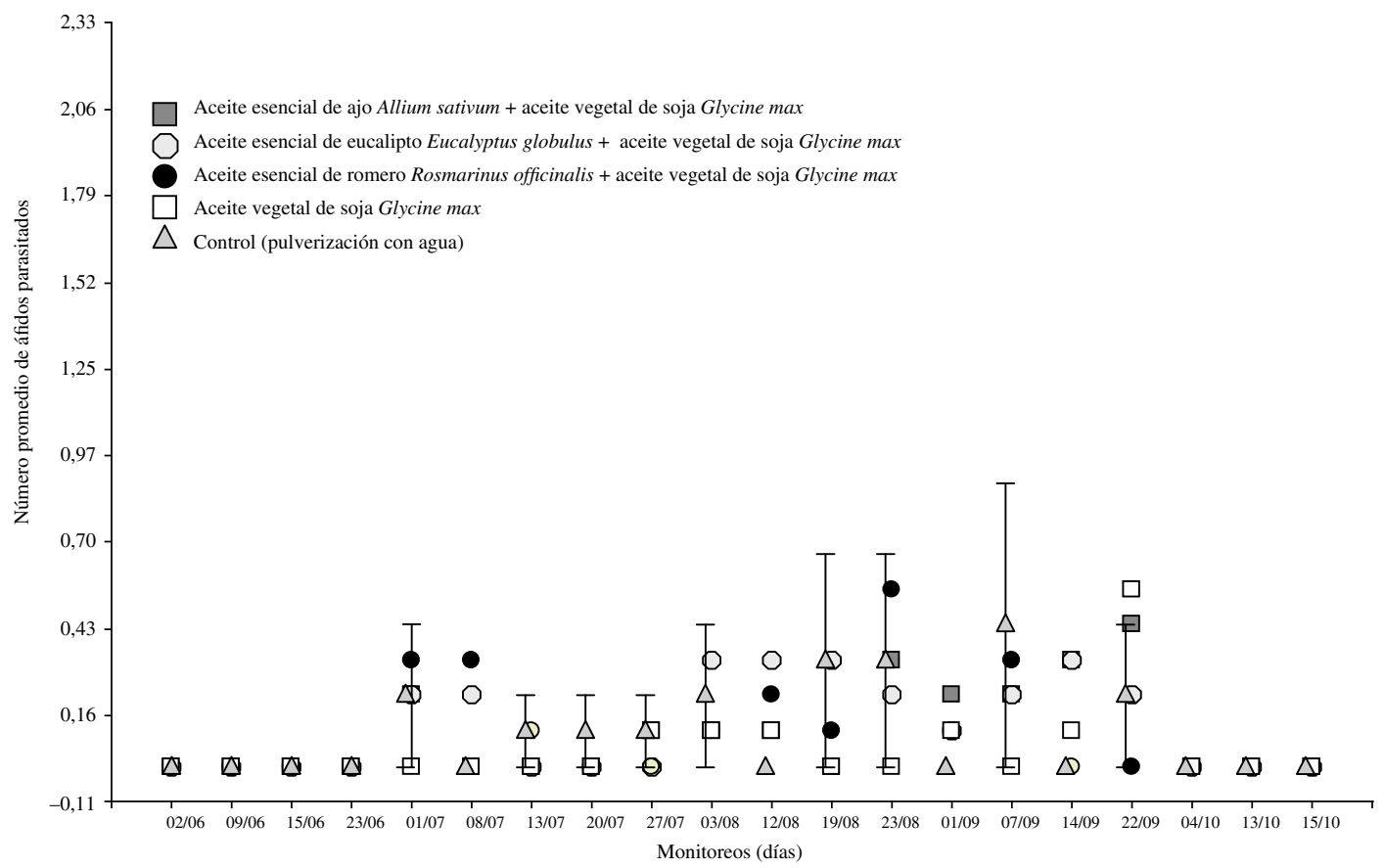

Figura 4. Población de áfidos parasitados sobre distintos tratamientos en invernadero no biosolarizado.

Análisis de la varianza

\begin{tabular}{lrrrr}
\hline & numDF & denDF & F-value & p-value \\
\hline (Intercept) & 1 & 190 & 7195,24 & $<0,0001$ \\
Tratamiento & 4 & 10 & 0,20 & 0,9308 \\
Semana & 19 & 190 & 2,42 & 0,0013 \\
Tratamiento: semana & 76 & 190 & 0,57 & 0,9969 \\
\hline
\end{tabular}

Del análisis realizado se determinó que no hay interacción estadísticamente significativa entre tratamiento y semana $(p=0,9969)$, tampoco diferencias entre tratamientos $(\mathrm{p}=0,9308)$; sin embargo, existe efecto del tiempo $(\mathrm{p}=0,0013)$.

Medias ajustadas y errores estándares para invernáculos

\begin{tabular}{ll}
\hline Invernáculos & Medias \\
\hline Invernáculo 1 & 5698,62 \\
Invernáculo 2 & 4661,04 \\
\hline
\end{tabular}

\section{Literatura Citada}

\section{Akhtar, Y. \& Isman, M. B.}

2004. Comparative growth inhibitory and antifeedant effects of plant extracts and pure allelochemicals on four phytophagous insect species. Journal of Applied Entomology, 128: 32-38.

Ahmadi, M.; Moharramipour, S. \& Zolfagharieh, H.

2007. Comparative fumigant toxicity of Rosmarinus officinalis and Artemisia sieberi. Conference of the IOBC WPRS Working Group on Integrated Protection of Stored Products. Poznan, Polonia, 65.
Batish, D.R.; Singh, H.P.; Kohli, R.K. \& Kaur, S.

2008. Eucalyptus essential oil as natural pesticide. For. Ecol. Manage., 256: 2166-2174.

Cabello García, T. \& Belda Suárez, J.

1994. Áfidos plaga (Homoptera: Aphididae) en cultivos hortícolas bajo plásticos. En: Moreno Vázquez R. (ed.). Sanidad Vegetal en la Horticultura Protegida. Cursos Superiores. Junta de Andalucía. Consejería de Agricultura y Pesca, pp. 157-177. 


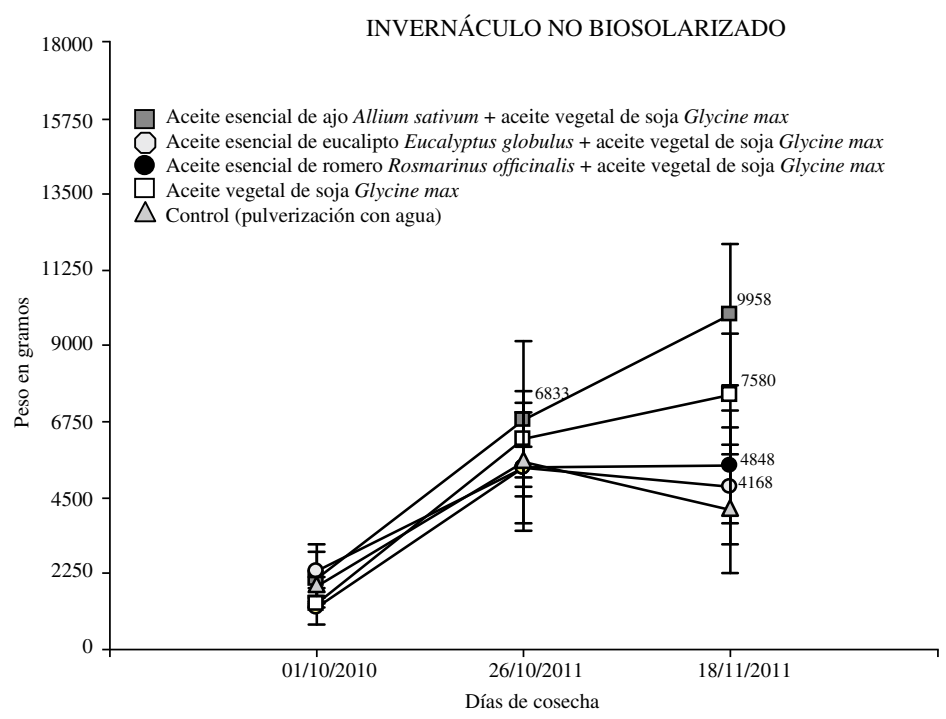

Figura 5. Peso promedio de frutos en distintos tratamientos en distintas cosechas en invernadero no biosolarizado.

Modelos lineales generales y mixtos

Invernáculo biosolarizado

Pruebas de hipótesis secuenciales

\begin{tabular}{lcrr}
\hline & numDF & F-value & \multicolumn{1}{c}{ p-value } \\
\hline (Intercept) & 1 & 264,89 & $<0,0001$ \\
Tratamiento & 4 & 0,84 & 0,5094 \\
Tiempo & 2 & 11,80 & 0,0002 \\
Tratamiento: tiempo & 8 & 1,03 & 0,4392 \\
\hline
\end{tabular}

Capinera, J.L.

2005. Myzus persicae (Sulzer) (Insecta: Hemiptera: Aphididae). Publ. N ${ }^{\circ}$ EENY-222. University of Florida. http://www.ufl. edu/. (October 2005).

Fuentes Contreras, E.; Basoalto, E.; Sandoval, C.; Pavez, P.; Leal, C.; Burgos, R. y Muñoz, C.

2007. Evaluación de la eficacia, efecto residual y de volteo de aplicaciones en pretrasplante de insecticidas nicotinoides y mezclas de nicotinoide - priretroide para el control de Myzus persicae nicotianae (Hemiptera: Aphididae) en tabaco. Agricultura Técnica (Chile) 67 (1): 16-22.

Herron G.A.; Powis, K. \& Rophail, J.

2001. Insecticide resistance in Aphis gossypii Glover (Hemiptera: Aphididae), a serious threat to Australian cotton. Australian Journal of Entomology 40: 85-89.

Ho, S.H.; Koh, L.; Ma, Y.; Huang, Y. \& Sim, K.I.

1996. The oil of garlic, Allium sativum L. (Amaryllidaceae), as a potential grain protectant against Tribolium castaneum (Herbst) and Sitophilus zeamais Motsch. Postharvest Biology and Technology 9, pp. 41-48.

Hori, M.

1996. Settling inhibition and insecticidal activity of garlic and onion oils against Myzus persicae (Sulzer) (Homoptera:
Aphididae). Journal Applied Entomology and Zoology, 31 (4): 605-612.

Işı1k, M. \& Görür, G.

2009. Aphidicidial activity of seven essential oils aganist the cabbage aphid, Brevicoryne brassicae L. (Hemiptera: Aphididae). Munis Entomology \& Zoology, 4(2): 365-372. Isman, M.B.

1999. Pesticides based on plant essential oils. Pesticide Outlook, 10:68-72.

Miresmailli, S. \& Isman M.B.

2006. Efficacy and persistence of rosemary oil as an acaricide against twospotted spider mite (Acari: Tetranychidae) on greenhouse tomato. Journal of Economy Entomology, December; 99 (6):2015-23.

Park, H.M.; Kim, J.; Chang, K.S.; Kim, B. S.; Yang, Y.J.; Kim, G.H.; Shin, S.C. \& Park, K.I.L.

2011. Larvicidal Activity of Myrtaceae Essential Oils and Their Components against Aedes aegypti, Acute Toxicity on Daphnia magna, and Aqueous Residue. Journal of Medical Entomology, 48 (2): 405-410.

Polack, L.A. \& Mitidieri, M.

2002. Producción de tomate diferenciado. Protocolo preliminar de manejo integrado de plagas y enfermedades. Información 


\section{INVERNÁCULO BIOSOLARIZADO}

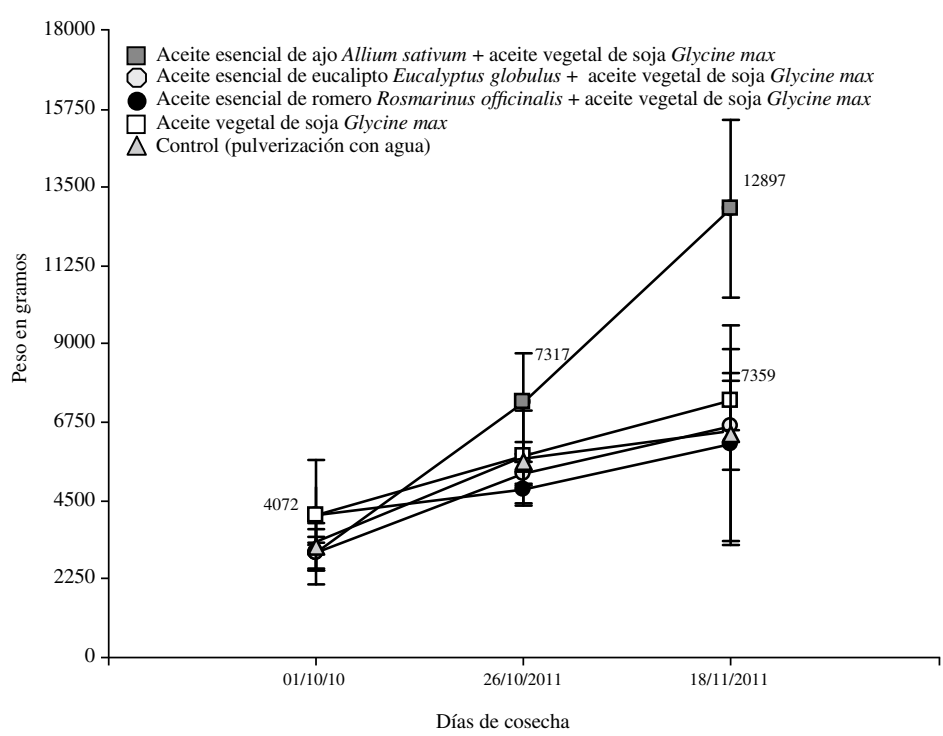

Figura 6. Peso promedio de frutos en distintos tratamientos en distintas cosechas en invernadero biosolarizado.

Modelos lineales generales y mixtos

Invernáculo sin biosolarizar

Pruebas de hipótesis secuenciales

\begin{tabular}{lcrr}
\hline & numDF & F-value & p-value \\
\hline (Intercept) & 1 & 117,64 & $<0,0001$ \\
Tratamiento & 4 & 0,77 & 0,5540 \\
Tiempo & 2 & 24,91 & 0,0001 \\
Tratamiento: Tiempo & 8 & 0,78 & 0,6269 \\
\hline
\end{tabular}

para Extensión Protección Vegetal № 20. Ediciones INTA. http://www.inta.gov.ar/sanpedro/info/doc/prv/ap_011. htm\#objec

Regnault-Roger, C.; Philogene, B. y Vincent, C.

2004. Biopesticidas de origen vegetal. 1ra ed., Editorial Mundi-Prensa. Madrid, España, pp. 20-38.

Ricci, E.M.; Padin, S.B.; Henning, C.; Ringuelet, J. \& Kahan A. 2010. Cineol para el manejo integrado de Myzus persicae y Brevicoryne brassicae en repollo. Bol. San.Veg. Plagas. 36: 37-43.

Sedy, K.A. \& Koschier, E.H.

2003. Bioactivity of carvacrol and thymol against Frankliniella occidentalis and Thrips tabaci. Journal of Applied Entomology 127: 313-316.

Smith, K.M.

1972. A text book of plant virus disease. 3th ed. New York, Academic Press. 684 p.

Syller, J.

1994. The effects of temperature on the availability and acquisition of Potato leaf roll luteovirus by Myzus persicae. Ann. Appl. Biol. 124: 141-149.

Topuz, E. \& Erler, F.

2007. Bioefficacy of some essential oils against the carmine spider mite, Tetranychus cinnabarinus. Fresenius Environmental Bulletin, 16B: 1498-1502.
Tunaz, H.; Kubilay, M. Er. \& Sikber, A. A.

2009. Fumigant toxicity of plant essential oils and selected monoterpenoid components against the adult german cockroach, Blattella germanica (L.) (Dictyoptera: Blattellidae) Turkish Journal of Agriculture and Forestry, 33 (2): 211-217.

Trumble, J.T.

2002. Caveat Emptor: Safety considerations for natural products used in arthropod control. American Entomologist, 48: 7-13.

Valério, E.; Cecílio, A. \& Mexia, A.

2005. Estratégias de Protecção Integrada para em cultura protegida de pimento. Record of proceedings of VII Encontro Nacional de Protecção Integrada-A Produção, Integrada e a Qualidade e Segurança Alimentar. Ma José Moreno da Cunha (coord.). Col. Práticas-Conhecimento-Pensamento, Ed. IPC (Instituto Politécnico de Coimbra). I1 (9): 98-105. Vasicek, A.; La Rossa, F. \& Paglioni, A.

2001. Aspectos biológicos y poblacionales de Aulacorthum solani, Myzus persicae, Macrosiphum euphorbiae (Homoptera: Aphidoidea) en pimiento (Capsicum annum L.). Bol. San. Veg. Plagas, 27: 439-446.

Zaouali, Y.; Bouzaine, T. \& Boussaid, M.

2010. Essential oils composition in two Rosmarinus officinalis L. varieties and incidence for antimicrobial and antioxidant activities. Food Chem. Toxicol. 48: 3144-3152. 